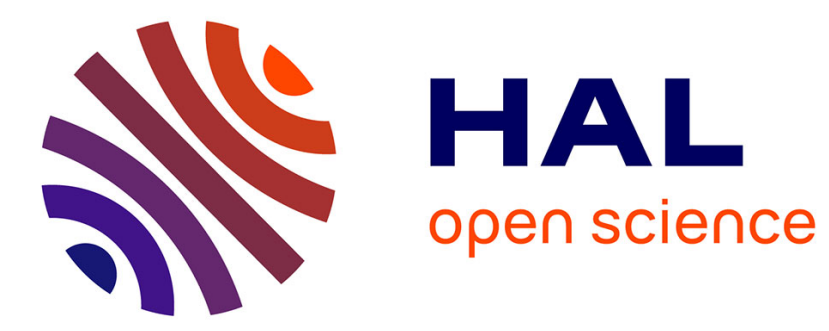

\title{
Amplitudes of the logarithmic singularities for the four-dimensional critical behavior
}

\author{
E. Brézin
}

\section{To cite this version:}

E. Brézin. Amplitudes of the logarithmic singularities for the four-dimensional critical behavior. Journal de Physique Lettres, 1975, 36 (3), pp.51-53. 10.1051/jphyslet:0197500360305100 . jpa00231152

\section{HAL Id: jpa-00231152 https://hal.science/jpa-00231152}

Submitted on 1 Jan 1975

HAL is a multi-disciplinary open access archive for the deposit and dissemination of scientific research documents, whether they are published or not. The documents may come from teaching and research institutions in France or abroad, or from public or private research centers.
L'archive ouverte pluridisciplinaire HAL, est destinée au dépôt et à la diffusion de documents scientifiques de niveau recherche, publiés ou non, émanant des établissements d'enseignement et de recherche français ou étrangers, des laboratoires publics ou privés. 


\title{
AMPLITUDES OF THE LOGARITHMIC SINGULARITIES FOR THE FOUR-DIMENSIONAL CRITICAL BEHAVIOR
}

\author{
E. BRÉZIN $(*)$ \\ Jefferson Physical Laboratory, Harvard University \\ Cambridge, Massachusetts 02138, U.S.A.
}

(Reçu le 23 décembre 1974, accepté le 9 janvier 1975)

\begin{abstract}
Résumé. - A quatre dimensions la théorie du champ moléculaire n'est pas valable car il y a violation des propriétés d'invariance d'échelle par des puissances de termes logarithmiques. C'est pourquoi il n'est pas correct de prendre la limite $\varepsilon=0$ d'une théorie à $4-\varepsilon$ dimensions. Cependant les rapports des coefficients des singularités logarithmiques sont, dans la plupart des cas, obtenus correctement par cette limite. Néanmoins, il est parfois nécessaire d'être prudent et un exemple est donné d'un rapport qui n'est pas égal à sa valeur de champ moyen.

Abstract. - In four dimensions mean field theory is not valid since scaling is violated by powers of logarithms. Therefore the theory is not the $\varepsilon=0$ limit of the $4-\varepsilon$ dimensional theory. However ratios of critical amplitudes are in most cases correctly described by this limit. In some cases, it is nevertheless necessary to be cautious, and an example is provided of a ratio which does not coincide with its mean field value.
\end{abstract}

Several years ago Larkin and Khmel'nitskii [1] discovered that in four dimensions the critical behavior was classical apart from logarithmic factors. They also showed that this four-dimensional problem was relevant for the discussion of the critical behavior of a three-dimensional uniaxial ferroelectric. This was further supported by A. Aharony's study [2] of uniaxial magnets with dipolar interactions. This $d=4$ problem is therefore more than a mere mathematical exercise. Most calculations have been done by the original authors; the renormalization group (R.G.) techniques developed later by $\mathrm{K}$. Wilson [3] provide a more transparent approach to this problem, and they have been applied either through recursion formulae [4] or through field theoretical methods [5]. In all cases, one discovers that the effective coupling constant which governs the long-distance behavior of the theory is small like the inverse of the logarithm of the correlation length. Thus the problem may be solved by using the renormalization group equations and perturbation theory.

The specific problem that we have studied here concerns the amplitude of the singularities. Let us discuss for definiteness the example of the specific heat ratio. When $d$ is less than four we know that the singular behavior of the specific heat [6] may be described in terms of $t=\left(T-T_{\mathrm{c}}\right)$ as,

$$
C= \begin{cases}A_{+} t^{-\alpha} & t>0 \\ A_{-}(-t)^{-\alpha^{\prime}} & t<0\end{cases}
$$

$\left({ }^{*}\right)$ On leave of absence from the Service de Physique Théorique, Saclay, B.P. 2, 91190, Gif-sur-Yvette, France. with

$$
\alpha=\alpha^{\prime}=\frac{(4-n)}{2(n+8)} \varepsilon+0\left(\varepsilon^{2}\right)
$$

and

$$
\frac{A_{+}}{A_{-}}=\frac{n}{4} 2^{\alpha}(1+\varepsilon)+0\left(\varepsilon^{2}\right)
$$

in which as usual $\varepsilon=4-d$ and $n$ is the number of components of the order parameter. In four dimensions the corresponding formulae read

$$
C= \begin{cases}\tilde{A}_{+}|\ln t|^{\frac{4-n}{n+8}} & t>0 \\ \tilde{A}_{-}|\ln (-t)|^{\frac{4-n}{n+8}} & -t<0\end{cases}
$$

and thus eq. (4) is not the $\varepsilon=0$ limit of eq. (1). (Even the regular terms would not help; in order to obtain the limit correctly it would be necessary to include in eq. (1) the correction-to-scaling terms). The question we are concerned with is the ratio $\tilde{A}_{+} / \tilde{A}_{-}$. Though the limit of eq. (4) is not eq. (1) it will be shown below that

$$
\tilde{A}_{+} \mid \tilde{A}_{-}=\lim _{\varepsilon \rightarrow 0} A_{+} / A_{-}=n / 4 .
$$

This property holds in other cases, but it will be argued that it is not always true and a specific counterexample will be provided.

1. Calculation of the free energy in zero field. - It has been shown in reference [5] that the free energy $\Gamma(M, t, g)$ in which $M$ is the magnetization, and $g$ 
the coupling constant of the four-spin interaction, satisfies an R.G. equation whose solution is

$$
\Gamma(M, t, g)=\lambda^{4} \Gamma\left(\frac{M(\lambda)}{\lambda}, \frac{t(\lambda)}{\lambda^{2}}, g(\lambda)\right)
$$

in which we have used dilatation-dependent variables defined by the equations

$$
\begin{aligned}
\ln \lambda & =\int_{g}^{g(\lambda)} \frac{\mathrm{d} g^{\prime}}{\beta\left(g^{\prime}\right)} \\
\frac{t(\lambda)}{t} & =\exp -\int_{g}^{g(\lambda)} \frac{\frac{1}{v\left(g^{\prime}\right)}-2}{\beta\left(g^{\prime}\right)} \mathrm{d} g^{\prime} \\
\frac{M(\lambda)}{M} & =\exp -\frac{1}{2} \int_{g}^{g(\lambda)} \frac{\eta\left(g^{\prime}\right)}{\beta\left(g^{\prime}\right)} \mathrm{d} g^{\prime}
\end{aligned}
$$

The functions $\beta(g), \eta(g), v(g)$ can be determined from a perturbation theory [7]. They are related by the following expressions.

$$
\begin{aligned}
\beta(g) & =\frac{n+8}{6} g^{2}+0\left(g^{3}\right) \\
\frac{1}{v(g)}-2 & =-\frac{n+2}{6} g+0\left(g^{2}\right) \\
\eta(g) & =\frac{n+2}{72} g^{2}+0\left(g^{3}\right)
\end{aligned}
$$

Up to now, $\lambda$ has been an arbitrary dilatation parameter in eq. (6). If $\lambda$ goes to zero, and we shall see later why it does, integration of eq. (7)-(12) gives at leading order

$$
\begin{aligned}
g(\lambda) & =\frac{6}{n+8}|\ln \lambda|^{-1} \\
t(\lambda) & =a t|\ln \lambda|^{-\left(\frac{n+2}{n+8}\right)} \\
M(\lambda) & =b M
\end{aligned}
$$

in which $a$ and $b$ are two constants which depend on the value of the initial coupling constant $g$.

1.1 Behavior ABOVE $T_{\mathrm{c}}$. We chose $\lambda$ so that

$$
t(\lambda) / \lambda^{2}=1 \text {. }
$$

Together with eq. (14) this means that in the critical region $\lambda$ goes to zero as

$$
\lambda^{2}=a t\left|\frac{1}{2} \ln t\right|^{-\left(\frac{n+2}{n+8}\right)} .
$$

Thus $g(\lambda)$ goes to zero when $t$ is small. Consequently, from eq. (6) we see that it is sufficient to know the free-energy for $g$ small in order to determine the critical free-energy completely. Perturbation theory gives for small $g$

$$
\Gamma_{\text {pert. th. }}(M, t, g)=\frac{1}{2} t M^{2}+g \frac{M^{4}}{4 !}-\frac{3 n}{2(4-n)} \frac{t^{2}}{g} \text {. }
$$

The last term of the r.h.s. of eq. (18) is a regular term, which is generated by the non-multiplicatively renormalizable character of the energy-energy correlation function [5].

We therefore conclude that above $T_{\mathrm{c}}$ in zero-field the free-energy behaves as

$$
\Gamma(M=0, t, g)=-\frac{3 n}{2(4-n)} \frac{\lambda^{4}}{g(\lambda)}
$$

or using eq. (17)

$$
\begin{array}{r}
C=-\frac{\partial^{2} \Gamma}{\partial t^{2}}=\frac{3 n}{4-n} \frac{a^{2}}{6}(n+8)\left|\frac{1}{2} \ln t\right|_{t>0}^{\frac{4-n}{n+8}}, \\
t>
\end{array}
$$

1.2 BehaVIOR Below $T_{\mathrm{c}}$. - Since $t$ is negative we now choose $\lambda$ so that

$$
\frac{t(\lambda)}{\lambda^{2}}=-1
$$

This has the effect of replacing $t$ by $(-t)$ in eq. (17). We must also determine the free-energy in zero-field below $T_{\mathrm{c}}$ in perturbation theory. Solving the equation $(\partial \Gamma / \partial M)_{\text {pert. th. }}=0$ for $t<0$ we obtain

$$
M^{2}=-(6 t / g)
$$

and thus

$$
\Gamma_{\text {pert. th. }}(M, t<0, g)_{H=0}=-\frac{3}{2} \frac{4}{4-n} \frac{t^{2}}{g} \text {. }
$$

Therefore eq. (19) is replaced by

$$
\Gamma(M, t<0, g)_{H=0}=-\frac{3}{2} \frac{4}{4-n} \frac{\lambda^{4}}{g(\lambda)} .
$$

We therefore end up with

$$
C=\frac{3.4}{4-n} \frac{a^{2}}{6}(n+8)\left|\frac{1}{2} \ln \right| t||^{\frac{4-n}{n+8}} \quad t<0
$$

2. Other amplitude ratios. - Similar calculations may be done for other ratios - for instance the magnetic susceptibility $(n=1)$ behaves as

$$
\chi= \begin{cases}\tilde{C}+\frac{1}{t}|\ln t|^{1 / 3}, & t>0 \\ \tilde{C}-\frac{1}{t}|\ln | t||^{1 / 3}, & t<0\end{cases}
$$

with

$$
\frac{\tilde{C}_{+}}{\tilde{C}_{-}}=\lim _{\varepsilon \rightarrow 0} \frac{C_{+}}{C_{-}}=2 .
$$

However, let us consider the ratio $\tilde{Q}_{1}$ defined in the 
following way : if we define the amplitudes $\tilde{C}_{+}, \tilde{C}_{\mathrm{c}}$, and $\tilde{B}$ by

$\left\{\begin{array}{lll}\chi=\tilde{C}_{+} \frac{1}{t}|\ln t|^{\frac{n+2}{n+8}} & H=0, & t>0 \\ \chi=\tilde{C}_{\mathrm{c}} H^{-2 / 3}|\ln H|^{1 / 3} & & t=0 \\ M=\tilde{B}(-t)^{1 / 2}|\ln t|^{\frac{3}{n+8}} & H=0, & t<0\end{array}\right.$

then

$$
\tilde{Q}_{1}=\frac{\delta \tilde{C}_{\mathrm{c}}}{\left(\tilde{B}^{\delta-1} \tilde{C}^{+}\right)^{1 / \delta}}
$$

with $\delta=3$. The corresponding $Q_{1}$ is equal to one for mean field theory.

In order to calculate $\tilde{Q}_{1}$ we have to obtain the expression of the equation of state [5]. The derivative with respect to $M$ of eq. (6) gives

$$
H(M, t, g)=\lambda^{3} \frac{M(\lambda)}{M} H\left[\frac{M(\lambda)}{\lambda}, \frac{t(\lambda)}{\lambda^{2}}, g(\lambda)\right] .
$$

By the same arguments as above, we know that it is sufficient now to compute $H$ in perturbation theory :

$$
H_{\text {pert. th. }}=t M+g M^{3} / 6 .
$$

However we now have to choose $\lambda$ and it is necessary to distinguish between two regimes i) $M^{2} /|t|$ is not infinitesimally small and then we can fix $\lambda$ by the condition

$$
M(\lambda) / \lambda=1
$$

ii) above $T_{\mathrm{c}}$ when $H$ goes to zero, $M^{2} /|t|$ is small and we have to fix $\lambda$ by the condition

$$
t(\lambda) / \lambda^{2}=1
$$

The same algebra leads to the results :

$H(M, t, g)=a b^{2} t M|\ln M|^{-\left(\frac{n+2}{n+8}\right)}+\frac{b^{4}}{n+8} \frac{M^{3}}{|\ln M|}$ except when $M^{2} / t \ll 1$, in which case we must use the condition (31) and the result is

$$
\begin{array}{r}
H(M, t, g)=a b^{2} t M\left|\frac{1}{2} \ln t\right|^{-\left(\frac{n+2}{n+8}\right)}+ \\
+\frac{b^{4}}{n+8} \frac{M^{3}}{\left|\frac{1}{2} \ln t\right|} .
\end{array}
$$

From eq. (32) we find

$$
\tilde{C}_{\mathrm{c}}=\frac{1}{3}\left(\frac{n+8}{3 b^{4}}\right)^{1 / 3}
$$

and

$$
\tilde{B}^{2}=\frac{a}{b^{2}}(n+8) 2^{-\left(\frac{6}{n+8}\right)}
$$

and from eq. (33)

$$
\tilde{C}_{+}=\frac{1}{a b^{2}} 2^{-\left(\frac{n+2}{n+8}\right)}
$$

We can now compute $\tilde{Q}_{1}$; the result is

$$
\tilde{Q}_{1}=\left(\frac{2}{3}\right)^{1 / 3}
$$

instead of one. This means that if logarithmic corrections are present, it is necessary to be careful if one considers universal ratios concerning amplitudes governing logarithmic singularities in different variables.

Acknowledgments. - This problem originated from a discussion with Professor P. Hohenberg. It is also a pleasure to thank Dr. A. Aharony for informative discussions.

\section{References}

[1] Larkin, A. and Khmel'NITSKII, D., Sh. Eksp. Theor. Fiz. 56 (1969) 2087 [Sov. Phys. JETP 29 (1969) 1123].

[2] Aharony, A., Phys. Rev. B 8 (1973) 3363.

[3] WILSON, K. and KoGUT, J., The renormalization group and the $\varepsilon$ expansion, Phys. Rep. (in press).

[4] Wegner, F. and Riedel, E., Phys. Rev. B 7 (1973) 248.

[5] Brézin, E., Le Guillou, J. C. and Zinn-Justin, J., Phys. Rev. D 8 (1973) 2418
For a review see Field theoretical approach to critical behavior (Saclay preprint).

[6] Brézin, E., Le Guillou, J. C. and Zinn-Justin, J., Phys. Lett. 47A (1974) 285.

[7] Brézin, E., Le Guillou, J. C. and Zinn-Justin, J., Phys. Rev. D 9 (1974) 1121. 